\title{
Verzeichnis der von Dr. F. Eichelbaum im Jahre 1903 in Deutsch-Ostafrika gesammelten Scydmaeniden (Col.).
}

\author{
Von Edm. Reitter in Paskau (Mähren).
}

Herr Dr. med. F. Eichelbaum war so freundlich, mich mit der Bearbeitung der auf seinen Reisen im Jahre 1903 in DeutschOstafrika gesammelten Scydmaeniden zu beauftragen. Indem ich das Ergebnis seiner erfolgreichen Sammlungen hier mitteile, danke ich demselben für die hochberzige Überlassung der zahlreichen Unica für meine Collection.

Amani ist die biologisch-landwirtschaftliche Station im OstUsambaragebirge, $920 \mathrm{~m}$ hoch, 80 Kilometer von der Küste entfernt. Bomole ist der höchste Berg desselben Gebirgszuges, $1100 \mathrm{~m}$.

Fast die ganze Scydmaeniden-Ausbeute stammt von diesen Lokalitäten, einige Arten sind auch in den Hafenplätzen Dar-es-Salâm und Tanga erbeutet worden.

\section{Gen. Cephennomicrus nov.}

Neue Gattung, nahe mit Cephennium verwandt. Von dieser durch die äußerst kleine. kurze und breite Gestalt, große Augen, elfgliederige Fühler mit großer zweigliedriger Keule und die Skulptur des Halsschildes abweichend. Am Thorax kommt bei manchen Cephenniumartigen Tieren ein Grübchen in den Hinterwinkeln vor, nicht aber solche auf der Scheibe. Bei vorliegender Gattung zeigt der annähernd viereckige Thorax außer einem Grübchen in den Hinterwinkeln noch zwei tiefe Grübchen vor dem Hinterrande. Wegen der zweigliedrigen Fühlerkeule zunächst neben Nanophthalmus Motsch. einzureihen.

Cephennomicrus perpunctillum n. sp. Parvulus, rufotestaceus, nitidus, minutissime, vix perspicue puberulus, antennis tenuibus, corporis dimidium attingentibus, clava abrupte biarticulata, articulo penultimo subquadrato, ultimo majore, apice appendiculato; capite parvo, oculis sat magnis nigris, granulatis, parum prominulis; prothorace haud punctato, leviter transversim quadrato, lateribus 
subparallelis, postice subtiliter marginatis, angulis posticis rectis, supra foreolatis, basi subtruncata, ante basin untrinque foveola profunde impresso; scutello sat magno, triangulari, in medio leviter foveolato; elytris thorace vix latioribus, breviter ovatis, convexis, vix perspicue punctatis, sublaevibus, basi late foreolatim impressis, callo humerali breviter lineatim prominulo; pedibus sat gracilibus, mediocriter elongatis, simplicibus. Long. $0.6 \mathrm{~mm}$ !

Kaum größer als die kleinste Ptinella, aber leicht kenntlich.

Ein einzelnes Exemplar wurde in der Zucht von ErotylidenLarven in Lehmerde vorgefunden. Amani, 30. Juli 1903.

Cephennium :an:ibaricum Schauf. B. 1889, 28, gehört wohl nicht in diese Gattung. Bei dieser Art sind die Fühler von der Nitte zur Spitze allmählich keulenförmig verdickt. Mir ist diese Art unbekannt.

\section{Genus Euconnus Thoms.}

I. Antennarum clava abrupte quadriarticulata.

A. Prothorax subtriangulatus, a basi ad apicem fortiter angustatus, ante basin transversim subsuleatus, sulea trifoveolata.

\section{Subgen. Napochus Thoms.}

Euconnus iconicus 11. sp. Parvus, subtilissime flavo-puberulus, antennis sat tenuibus, dimidium corporis superantibus, articulo secundo primo longiore, oblongo, $3--7$ parvis, subquadratis, clava quadriarticulata, parallela, articulis clavae praecedentibus duplo latioribus, tribus penultimis subaequalibus, non distincte transversis, transversosubquadratis, articulo ultimo latitudine vix longiore, apice leviter acuminato; capite triangulari, thorace basi parum angustiore, postice fortiter recte angustato, oculis in angulis anticis sitis, mediocribus, leviter prominulis, granulatis, temporibus magis longe pilosis; prothorace antrorsum fortiter subrecte angustato, coleopteris angustiore, basi in sulco tenui transverso trifoveolato, supra vix punctato; elytris ovatis, vix perspicue punctulatis, basi biimpressis, impressione externa majore, plica humerali brevi distincta; pedibus gracilibus, tibiis intermediis intus ante apicem obscure sinuatis. Long. $1 \mathrm{~mm}$.

Ein einzelnes Stück aus Waldlaub gesiebt vom Berge Bomole im Ost-Usambaragebirge am 11. Oktober 1903. Ist unseren kleinen Napochus-Arten ganz ähnlich, blaß rötlichgelb. 
B. Prothorax subrotundatus aut oblongo-subrotundatus, antice plus minusve rotundatus aut rotundatim angustatus.

a" Prothorax basi tri-aut quinque-foveolatus.

b" Femoribus fortiter inflatis, tibiis anticis apicem versus fortiter dilatatis, intus pone basin emarginatis et densissime flavo-tomentosis.

Euconnus torimanus n. sp. Castaneus, subtiliter fulvopubescens, antennis thoracis basin haud attingentibus, mediocribus, articulis $3-7$ parvis, subtransversis, clava quadriarticulata duplo latiore, parallela, articulis subaequalibus, tribus penultimis leviter transversis, ultimo latitudine vix longiore, apice obtuse acuminato; capite thorace angustiore, suboblongo, oculis granulatis, parum prominulis, temporibus magis dense pilosis, postice angustatis; prothorace coleopteris angustiore, subgloboso, latitudine parum longiore, antrorsum magis angustato, vix punctato, basi quinquefoveolato; elytris late et breviter ovatis, dense subtiliter punctatis et subtiliter puberulis, basi impressa, impressione subbifoveolata, extus humeris obtuse fortiter elevatis; pedibus robustis, tibiis leviter arcuatis, posterioribus sensim tenuioribus, tarsis simplicibus. Long. $1.75 \mathrm{~mm}$.

Im ganzen vier Stück um Amani im Dezember 1903 gefunden, darunter eins im Oktober aus Waldlaub vom Berge Bomole gesiebt. b' Femoribus haud fortiter inflatis, tibiis anticis simplicibus.

Durch Schaufuß wurden einige Arten, die hieher gehören möchten, aus Zanzibar beschrieben, aber von Dr. Eichelbaum vom Festlande nicht mitgebracht.

a' Prothorax bi- aut quadrifoveolatus.

c" Prothorax ante basin vix transversim suleatus, in medio basi haud plicatus. d" Prothorax sublaevigatus, vix perspicue punctatus. Körper klein, zirka $1 \mathrm{~mm}$.

Euconnus rubiginosus Schauf., B. 1889, 15, aus Arabien beschrieben. Auf diese Art paßt ziemlich ein schlecht erhaltenes Exemplar vom Berge Bomole, welches am 13. Oktober gesiebt wurde, nur ist die Gestalt gedrungener und dürften weitere Stücke eine besondere Art ergeben.

Eucon. biocellatus Schauf. 1. c. 17, zwei Stück aus Waldlaub des Berges Bomole am 11. und 13. Oktober 1903 gesiebt.

d' Elytris prothoraceque distincte punctatis. Körper dunkelbraun, stark, etwas rauh behaart, von zirka $2 \mathrm{~mm}$ Länge. Die Mittelschienen innen im letzten Drittel breit und flach ausgebuchtet und mit gelben kurzem Haartoment besetzt.

Euconmus (Glaphostoma) cribricollis Schauf. B. 1889, 2. Amani, zwei Stücke, 9-12, 1903. 
Auf diese Art wurde von L. W. Schaufuß das Genus Glaphostoma (l. c.) aufgestellt, auf Grund der zwei letzten Tasterglieder, die ich aber von anderen Eucommus-Arten nicht verschieden finden kann; auch der sonstige Bau des Körpers gibt zur generischen Abtrennung keinen Anlaß.

$\mathrm{c}^{\prime}$ Prothorax ante basin transversim suleatus, sulco in medio et ad latera plica distincta ornato, untrinque obsolete bifoveolato.

Euconnus neuraphiformis n. sp. Parvulus, rufus, nitidus, subtilissime puberulus, antennis tenuibus, dimidium corporis fere superantibus, articulis parvis $3-6$ subquadratis aut oblongo-subquadratis, clava quadriarticulata parum latiore, subparallela, articulis subglobosis, indistincte transversis, articulo ultimo haud latiore sed paulo longiore; eapite subrotundato, vix punctato, thorace minus angustiore, oculis mediocribus, distinctis, vix prominulis, temporibus postice angustatis; prothorace latitudine parum longiore, coleopteris angustiore, antice globoso, rotundato, postice fere parallelo, vix punctato, ante basin transversim sulcato, triplicato et obsolete quadrifoveolato; elytris breviter ovalibus, subtilissime, vix perspicue punctulatis, basi impressis, impressione antice parum bifoveolata; pedibus simplicibus. Long. $1 \mathrm{~mm}$.

Amani. Am 13. Oktober 1903 aus Waldlaub des Berges Bomole in einem Stück gesiebt.

II. Antennarum clava abrupte quinquearticulata.

\section{Subgen. Eupentarius nov.}

Euconnus grandiclavis n. sp. Rufo-ferrugineus, antennis palpis pedibusque parum dilutioribus, sat longe et dense fulvopubescens, antennis robustis, dimidium corporis fere attingentibus, articulo $2^{0}$ oblongo-subquadrato, $3-6$ parvulis transversis, clava abrupte quinquearticulata, praecedentibus articulis fere plus quam duplo latiore, articulo clavae primo majore quam secundo, introrsum angulo apicali oblique truncato, secundo sequentibus paullo minore, duobus penultimis subtransversis, ultimo minus longiore, acuminato; capite cum oculis mediocribus, granulatis, parum prominulis, vix thorace angustiore, latitudine fere longiore, temporibus postice angustatis; prothorace subgloboso, latitudine parum longiore, vix punctato, ante basin tripunctato, puncto in medio parvulo, lateribus ante basin obsolete foreolato; elytris latis, valde ampliatis, breviter ovatis, 
subtiliter punctatis, plica humerali obtusa brevi fortiter elevata, pedibus gracilibus, fere simplicibus. Long. $1.8 \mathrm{~mm}$.

Amani; ein einziges Exemplar vom Dezember 1903.

III. Antennarum clava abrupte triarticulata.

Subgen. Scydmaenites Croissandeau.

Euconnus iners n. sp. Oblongus, castaneus, antennarum clava, palpis pedibusque dilute rufis, subtiliter fulvo-pubescens; antennis mediocribus, thoracis basin vix attingentibus, articulis 3-8 parvulis subtransversis; clava abrupte triarticulata, aequilata, articulis 2 penultimis leviter sed distincte transrersis, articulo ultimo oblongo-ovato, longitudine duobus praecedentibus conjunctis aequalibus; capite subrotundato, ferrugineo, thorace parum angustiore, postice rotundato, oculis haud preminulis; prothorace coleopteris parum angustiore, subgloboso, latitudine longiore, convexo, nitido, vix punctato, sublaevi, ante basin obsolete transversim subsulcato, in sulco indistincte foveolato; elytris breviter ellipticis, subtilissime disperse punctulatis, fere glabris, subtiliter puberulis; pedibus simplicibus. Long. $1.5 \mathrm{~mm}$.

Ein einzelnes Exemplar aus Waldlaub des Berges Bomole, in Ost-Usambaragebirge am 11. Oktober 1903 gesiebt.

\section{Genus Scydmaenus Latr.}

\section{Syn. Eumicrus Lap.}

I. Elytris basi foveolatim impressis. Prothorax basi distincte quadrifoveolatus.

(Scydmaenus in spe.)

A. Antennis elongatis, articulo quinto latidudine duplo longiore. Species majores Long. 2-3 $\mathrm{mm}$.

a" Prothorax foveolis quatuor antebasalibus parvis, punctiformibus.

Scydm. scutellatus Schauf. B. 1889, 33. - Amani; vom September bis Dezember einzeln gesammelt. Von den nachfolgenden durch die kleineren punktförmigen Basalgrübchen des Halsschildes abweichend, welche in gleichen Entfernungen stehen.

Scydmaenus Hyrtacides n. sp. Oblongus, rufo-testaceus, dense subtiliter flaveque puberulus, antennis elongatis, sat robustis, articulo 2 et 6 subquadratis, latitudine minus longioribus, 3 et 4 
leviter oblongis, articulis 7 et 8 parvis, transversis, articulo $5^{0}$ elongato, latitudine fere duplo longitudine, clava triarticulata, articulis duobus penultimis quadratis, ultimo oblongo-ovato, majore, longitudine duobus praecedentibus aequali; capite thorace angustiore, subrotundato, vix perspicue punctulato, temporibus postice valde angustatis; prothorace oblongo-0vato, ante medium ad apicem fortiter, ad basin leviter angustato, vix punctato, foveolis parvulis, punctiformibus leviter impressis, exterioribus fere minoribus; elytris ovalibus; sat convexis, thorace latioribus, dense subtilissime punctulatis et magis dense puberulis, pedibus simplicibus. Long. $2.5 \mathrm{~mm}$.

Dem $q$ von Eichelbaumi äußerst ähnlich; besonders durch die Form, Größe, Färbung und die seidenartige, nicht ganz anliegende Behaarung und die äußerst feine Punktur der Flügeldecken abweichend. Von scutellatus durch schlankeren Körper, hellere Farbe, dichtere und feine Punktur der Flügeldecken und den längeren, mehr gerundeten Kopf verschieden.

A mani, September bis Dezember 1903, ein einzelnes Exemplar.

a' Prothorax foveolis antebasalibus majoribus subaequalibus et fortiter impressis.

b" Prothorax oblongo-subeylindricus.

Scydm. duricollis n. sp. Subtiliter fulvo-pubescens, piceus, nitidus, antennis medium corporis fere superantibus, articulis basalibus oblongis, 7 et $8^{0}$ subtransversis, clava triarticulata, articulis 9 et 10 subquadratis, articulo ultimo majore, ovato, subacuminato; capite subquadrato, sat parvulo, thorace angustiore, temporibus postice paullo angustatis, angulis rotundatis, oculis parvis, haud prominulis, supra vix perspicue punctulato, fere laevi; prothorace valido, subcylindrico, latitudine evidenter longiore, angulis rotundatis, supra convexo, parce vix perspicue punctulato, foveolis sat magnis subaequalibus ante basin impressis; elytris thorace latioribus, breviter ovalibus, magis distincte pubescentibus, subtiliter dense punctatis, basi late foreolatis. Long. $2 \cdot 8 \mathrm{~mm}$.

Mas. Femoribus magis inflatis, tarsis anterioribus et intermediis leviter dilatatis.

Der Thorax ist fast cylindrisch, an den Seiten wenig gerundet, in oder dicht vor der Mitte am breitesten, viel länger als breit, vorne nicht allmählig, sondern plötzlich zum Halse verengt.

Von scutellatus Schauf., dem er täuschend ähnlich ist, durch den robusteren cylindrischen Thorax und seine größeren Basalgrübchen abweichend. 
A mani. Zwei Exemplare, September bis Dezember; ein Exemplar von Bomole, 920 resp. $1100 \mathrm{~m}$ über dem Meere; Tanga, ein Exemplar im August 1903.

b' Prothorax oblongo-subovatus.

e" Tibiis posticis maris vix calcaratis.

Scydmaenus amaniensis n. sp. Sc. scutellato simillimus, sed minor, antennis paullo brevioribus, articulo quinto latitudine vix duplo longiore, capite transversim subquadrato, temporibus subparallelis, postice subtruncato, prothorace subovato, parum elongato, fere inconspicue punctulato, foveolis antebasalibus majoribus, subaequalibus, elytris breviter ovatis, subtiliter punctulatis. Long. $2 \mathrm{~mm}$. Mas. Tarsis quatuor anterioribus leviter dilatatis.

Einfarbig braunrot, fein rotbraun, geneigt behaart. Von dem sehr ähnlichen scutellatus Schauf. durch kleinere Körperform, kürzeren Thorax und mehr parallele Schläfen des Kopfes, sowie durch die größeren Basalgrübchen des Halsschildes abweichend. Der letatere vor der Mitte am breitesten.

Amani, September bis Dezember gesiebt, einige aus Laublagen des Waldes am Berge Bomole im Ost-Usambragebirge gesiebt.

Scydmaenus spathifer n. sp. Breviter fulvopubescens, castaneus, nitidus, antennarum articulis basalibus oblongis, 6 oblongoquadrato, 7 et 8 transversis, clava triarticulata, magna, articulis duobus penultimis subquadratis, ultimo majore, oblongo, subacuminato; capite thorace fere dimidio angustiore, parce subtilissime punctato, angulis rotundatis, oculis parvis, haud prominulis; prothorace coleopteris angustiore, convexo, oblongo-ovato, vix perspicue punctulato, fere sublaevigato, foveolis antebasalibus quatuor impressis; elytris late ovatis, dense subtiliter punctatis, foveola lata basali parum obsoleta; pygidio dilutiore. Long. $3 \mathrm{~mm}$.

Mas. Antennarum articulo penultimo subtus excarato; tibiis posticis sensim leviter curvatis, introrsum ante apicem leviter dilatatis; tarsis anticis levissime dilatatis.

Etwas größer als $S c$. scutellatus, dem er sehr ähnlich ist, aber die Basalgrübchen des Halsschildes größer und durch die männlichen Geschlechtsauszeichnungen abweichend. Das vorletzte Fühlerglied des $\sigma^{\nearrow}$ ist in gewisser Richtung innen ausgehöhlt, das letzte fast dreieckige Glied sitzt am inneren Spitzenwinkel des vorletzten auf, was bei einer Drehung des Objektes und der Ansicht ron der 
Seite bemerkbar wird. Beim $Q$ ist das vorletzte Glied nicht ausgehöhlt, aber das letzte Glied sitzt ebenfalls am inneren Spitzenwinkel des vorletzten auf. Die Hinterschienen des $\sigma^{x}$ sind leicht gebogen, das apicale, innere Drittel ist deutlich verbreitert, alle Ecken der Erweiterung abgerundet.

Nur $\sigma^{\Upsilon}$ q bei Amani im September und Dezember 1903 gesiebt.

e' Tibiis posticis maris intus distincte calearatıs.

Scydmaenus Eichelbaumi n. sp. Dilute rufus, dense subtiliter fulvo-puberulus, antennis elongatis, articulis $1-6$ oblongis, quinto elongato, sexto latitudine sesquilongiore, 7 et 8 parvis, subtransveris, clava magna, triarticulata, articulis duobus penultimis latitudine parum longioribus, ultimo elongato-orato, duobus penultimis fere longioribus, subtus in mare elongatim foveolato-excavato; capite thorace angustiore, subquadrato, angulis rotundatis, basi truncato, oculis parvis, haud prominulis; prothorace ovato, latitudine longiore, ante medium antrorsum magis quam basi angustato, ante basin 4 foveolis mediocribus impressis, elytris dense vix perspicue punctulatis, breviter ovatis, convexis. Long. $2 \cdot 3-2 \cdot 5 \mathrm{~mm}$.

Mas. Antennis longioribus, articulo ultimo elongato subtus longitudinaliter foreolatim excavato, tibiis intermediis intus ante apicem levissime late excisis, posticis apice intus breviter calcaratis; tarsis anticis intermediisque parum dilatatis.

Von scutellatus durch sehr feine und dichte Punktur, feinere und dichtere Behaarung und die Geschlechtscharaktere des or sehr abweichend.

Unter tiefen Laublagen der Wälder am Berge Bomole im Ost-Usambaragebirge am 11. und 13. Oktober 1904 von Dr. F. Eichelbaum in einiger Zahl gesiebt und dem Entdecker zu Ehren benannt.

B. Antennis mediocribus, articulo quinto latitudine vix aut parum longiore. Species minores. Long. $1.2-1.5 \mathrm{~mm}$.

Scydmaemus efflorescens Schauf. B. 1889, 36. - Amani, vom August bis Dezember einzeln gesiebt.

Scydm. nitidus Schauf. 1. c. 35. - Unter den vorigen ein Exemplar, das sich von diesem nur dadurch unterscheidet, daß die mittleren Basalgrübchen des Halsschildes etwas weiter auseinandergerückt sind. 
II. Elytris basi haud distincte foveolatim impressis. Prothorax basi haud aut indistincte foveolatus. (Subgen. Eustemmus Reitt.)

d" Capite thorace parum angustiore, baud longitudinaliter canaliculato.

Scydmaenus blandus Schauf. B. 1889, 34. Nur ein schlecht erhaltenes Exemplar dieser kleinen Art ron Tanga.

Scydmaenus ictericus n. sp. Oblongus, rufo-testaceus subtiliter flavopuberulus, antemnis sat tenuibus, dimidium corporis fere superantibus, articulis $2,3,4,6$ vix oblongis, subquadratis, articulo quinto evidenter oblongo, clava triarticulata, articulis duobus penultimis subquadratis, ultimo parum majore, ovato; capite thorace parum angustiore, subquadrato, sublaevi, angulis rotundatis, oculis parvulis, haud prominulis; prothorace ovato, leviter oblongo, haud punctato, basi vix foveolis impressis; elytris ovalibus, thorace parum latioribus, dense subtiliter punctulatis et magis puberulis, basi haud foveolatis, pedibus sat longis, simplicibus. Long. $1.8 \mathrm{~mm}$.

Ein einzelnes O rom Berge Bomole in Ost-Usambara aus Waldlaub gesiebt.

d' Capite thorace parum angustiore, subtransverso, profunde longitudinaliter canaliculato.

Scydmaenus delectus Schauf. B. 1889, 38. - Ausgezeichnet durch die tiefe Kopffurche, welche den Kopf in zwei Längsteile scheidet und den Thorax, welcher wenig schmäler ist als die Flügeldecken. seine Scheibe ist außerordentlich fein und dicht punktuliert. Ein einzelnes Stück im Culturgarten von Dar-es-Salâm in einem faulenden Polyporus lucidus gefunden. 


\section{$2 \mathrm{BHL}$ Biodiversity Heritage Library}

Reitter, Edmund. 1907. "Verzeichnis der von Dr. F. EICHELBAUM im Jahre 1903 in Deutsch-Ostafrika gesammelten Scydmaeniden (Col.)." Wiener entomologische Zeitung 26, 297-305. https://doi.org/10.5962/bhl.part.8894.

View This Item Online: https://www.biodiversitylibrary.org/item/45299

DOI: https://doi.org/10.5962/bhl.part.8894

Permalink: https://www.biodiversitylibrary.org/partpdf/8894

\section{Holding Institution}

Smithsonian Libraries

\section{Sponsored by}

Smithsonian

\section{Copyright \& Reuse}

Copyright Status: NOT_IN_COPYRIGHT

This document was created from content at the Biodiversity Heritage Library, the world's largest open access digital library for biodiversity literature and archives. Visit BHL at https://www.biodiversitylibrary.org. 